\title{
Natural history and predictors for progression of mild childhood obstructive sleep apnoea
}

\author{
A M Li, ${ }^{1}$ C T Au, ${ }^{1}$ S K Ng, ${ }^{2}$ V J Abdullah, ${ }^{2}$ C Ho, ${ }^{3}$ T F Fok, ${ }^{1}$ P C Ng, ${ }^{1}$ Y K Wing ${ }^{3}$
}

\section{See Editorial, p 4}

- Additional data are published online only at http://thorax.bmj. com/content/vol65/issue1

${ }^{1}$ Department of Paediatrics, Prince of Wales and Shatin Hospital, The Chinese University of Hong Kong, Shatin, Hong Kong; ${ }^{2}$ Department of Otorhinolaryngology, Prince of Wales and Shatin Hospital, The Chinese University of Hong Kong, Shatin, Hong Kong;

${ }^{3}$ Department of Psychiatry, Prince of Wales and Shatin Hospital, The Chinese University of Hong Kong, Shatin, Hong Kong

Correspondence to: Dr A M Li, Department of Paediatrics, Prince of Wales Hospital, The Chinese University of Hong Kong, Shatin, Hong

Kong; albertmli@cuhk.edu.hk

Received 21 May 2009 Accepted 11 September 2009

Published Online First

23 September 2009

\section{ABSTRACT}

Aims: The natural history of mild childhood obstructive sleep apnoea (OSA) was examined and factors associated with disease progression were identified.

Methods: Subjects were recruited from an epidemiological study which examined the prevalence of OSA in Chinese children aged $6-13$ years. The first 56 consecutive children identified with mild OSA (apnoeahypopnoea index 1-5) were invited for a repeat assessment 2 years after the diagnosis.

Results: 45 children participated in the follow-up study, in 13 of whom (29\%) the OSA was found to have worsened. Compared with those in whom OSA had not worsened, the worsened OSA group had a greater increase in waist circumference, a higher prevalence of large tonsils (occupying $\geqslant 50 \%$ of the airway) at both baseline and follow-up, and a higher prevalence of habitual snoring at both baseline and follow-up. The presence of large tonsils had a positive predictive value of $53 \%$ and a negative predictive value of $83 \%$ for worsening OSA over a 2-year period. Multivariate linear regression analysis showed that the change in obstructive apnoeahypopnoea index was associated with age at baseline ( $\beta$ $(\mathrm{SE})=-0.92(0.34), p=0.009)$, gender (male $=1$; female $=0)(\beta(S E)=4.69(1.29), p<0.001)$, presence of large tonsils at baseline ( $\beta$ (SE) $=4.36$ (1.24), $p=0.001)$, change in waist circumference $(\beta$ $(S E)=0.30(0.09), p=0.002)$ and persistently large tonsils ( $\beta(\mathrm{SE})=5.69(1.36), p<0.001)$ over the 2-year period.

Conclusions: Mild OSA in the majority of children does not resolve spontaneously. Subjects with tonsillar hypertrophy, especially boys, should be closely monitored to allow early detection of worsening OSA. Weight control should be stressed in the management of childhood OSA.

Childhood obstructive sleep apnoea (OSA) can lead to a number of important short- and long-term complications including hypertension, ventricular dysfunction, insulin resistance and neurocognitive deficits. ${ }^{1-5}$ The natural history of OSA is still uncertain. Prospective studies in adults suggest that weight gain and obesity are predictors of worsening of OSA. ${ }^{6-8}$ In contrast, convincing evidence on the natural history of paediatric OSA is lacking. Early studies were mostly based on subjective data from parent-reported questionnaires. ${ }^{10}$ Ali et al showed that over half of children with habitual snoring at 4-5 years of age no longer did so 2 years later. ${ }^{9}$ Urschitz et al performed a 1year follow-up study which also reported similar results. ${ }^{10}$ In addition, the investigators found that low maternal education, household smoking and loud snoring at baseline were predictors of persistent snoring in children. ${ }^{10}$ Two other studies in children suggested that most children with primary snoring did not progress to OSA over a course of several years. ${ }^{11}{ }^{12}$ To our knowledge, there is only one study that has examined the natural history of children with mild OSA..$^{13}$ In that study, three of seven children studied with mild OSA at the initial survey had significant disease progression. ${ }^{13}$ However, the investigators could not determine predictors for worsening of disease owing to the small sample size and lack of a control group.

Understanding the natural history of OSA and ascertainment of factors that are associated with disease worsening would allow a more scientific approach for future patient care. A progressive nature of the condition would mandate follow-up of mild cases who do not receive treatment, as well as potentially influencing treatment planning at an early stage. We aimed in this study to examine the natural history of mild childhood OSA and to investigate important risk factors that may influence disease progression.

\section{METHODS}

\section{Subjects and study design}

This was a prospective longitudinal follow-up study, an extension of our Childhood OSA Prevalence (COP) study which was initiated in 2003 with the aim of evaluating the prevalence of OSA in Hong Kong Chinese children aged 613 years. ${ }^{14}$ The COP study was a community-based study in which children were randomly selected from 13 primary schools in two districts in the territory. It was a two-phase study involving an OSA questionnaire screening followed by overnight polysomnography (PSG) confirmation and adenotonsillar size assessment by a paediatric otorhinolaryngologist. Subjects identified as having mild OSA (obstructive apnoea-hypopnoea index (OAHI) 1-5) and confirmed not to have received any treatment for their condition in the previous 2 years were invited to participate in this followup study. They completed the same OSA questionnaire and underwent the same set of investigations as previously.

\section{Questionnaire}

A validated sleep questionnaire based on parental reporting was given to each subject to obtain the following information:

- Snoring frequency and other OSA-related symptoms rated on a 5 -point rating scale $(0-$ 4) where $0=$ never, $1=$ rarely $(0-1$ night per month), 2 =sometimes (1-2 nights per month), $3=$ often (1-2 nights per week), $4=$ frequently ( $\geqslant 3$ nights per week). Habitual snoring was defined as snoring frequency 
scored 4. Similarly, other OSA-related symptoms were defined as positive only if a score of 4 was given.

- Clinical features: history of atopy and upper respiratory tract infection in the past 12 months.

- Socioeconomic and environmental factors: household smoking, family income and parental education. ${ }^{15}$

\section{Anthropometric measurements}

Anthropometric parameters including weight, height and waist circumference were measured on the day of PSG. Body mass index (BMI) and waist circumference were translated into $\mathrm{z}$ scores according to local references. ${ }^{16}{ }^{17}$ Obese children were defined as BMI z-score ( $z$-BMI) $>1.645$, corresponding to the 95th percentile (relative to age and gender).

\section{Polysomnography (PSG)}

All recruited children underwent initial and follow-up standard overnight PSG at a dedicated sleep laboratory with a CNS 1000P polygraph (CNS Inc, Chanhassen, Minnesota, USA) as described in our previous publication. ${ }^{18}$ All computerised sleep data were further manually scored by experienced PSG technologists and clinicians according to standardised criteria. ${ }^{19}$ We defined obstructive apnoea as absence of airflow with persistent respiratory effort lasting $\geqslant 2$ baseline breaths, irrespective of changes in oxygen saturation. Obstructive hypopnoea was defined as a reduction of $\geqslant 50 \%$ in the amplitude of the airflow signal with persistent respiratory effort. It was only quantified if $\geqslant 2$ baseline breaths and associated with oxygen desaturation of $\geqslant 3 \%$ and/or arousals. OAHI was defined as the total number of obstructive apnoeic and hypopnoeic episodes per hour of sleep. Oxygen desaturation index was defined as the total number of dips in arterial oxygen saturation (using Ohmeda 3700 pulse oximeter and measured by finger sensor, averaging time $3 \mathrm{~s}$ ) $\geqslant 3 \%$ per hour of sleep. Arousal was defined according to standard criteria. ${ }^{20}$ The total arousal index was defined as the total number of arousals per hour of sleep. Respiratory arousal index was defined as the number of arousals which followed apnoea, hypopnoea or flow limitation per hour of sleep.

Worsening of OSA was defined as an increase in OAHI greater than its measurement error. In our previous study which investigated the night-to-night variability of overnight sleep, 87 children underwent PSG on two consecutive nights. ${ }^{21}$ Thirty-six had an OAHI between 1 and 5 on the first night of the study. The subgroup had a common within-subject SD (sw) of 0.86 . According to Bland and Altman, ${ }^{22}$ the measurement error of OAHI was $1.96 \times \sqrt{2} \times \mathrm{sw}=2.38$. Hence, children with an increase in OAHI of $>2.38$ were classified into the group in whom OSA worsened while the others were classified as the referent group.

\section{Adenotonsillar size assessment}

The size of tonsils and adenoids was evaluated by endoscopic examination via a flexible fibrescope (Olympus P4). The examination was performed by an otorhinolaryngologist who was blind to the PSG result of the subjects. Tonsil size was reported as a percentage of the oropharyngeal airway while adenoid size was assessed as a percentage of the nasopharyngeal airway. A large tonsil or adenoid was defined as the soft tissue occupying $\geqslant 50 \%$ of the corresponding airway. Tonsils and adenoids were further classified as "persistently large" if they were large at both baseline and follow-up, as "persistently small" if they were small at both time points, as "shrunken" if they were large at baseline but not at follow-up, and as "enlarged" if they were small at baseline and became large at follow-up.

\section{Statistical analysis}

All the parametric and non-parametric data were expressed as mean (SD) and median (IOR), respectively. Paired $t$ tests, Wilcoxon signed rank tests and McNemar tests were used to examine intragroup differences between baseline and follow-up for parametric, non-parametric and dichotomous data, respectively. Student $t$ tests and $\chi^{2}$ tests (or Fisher exact tests) were used to detect between-group differences for continuous and categorical data, respectively. Linear regression analyses were performed to investigate factors that were associated with the change in OAHI. Two different multivariate models were developed to test if any baseline variables and if any changes in subject characteristics over the 2-year period were associated with the change in OAHI. Independent variables with $p<0.05$ in univariate analyses were put into the multivariate model with adjustment for age at baseline and gender. All analyses were performed using SPSS Version 13.0 (SPSS, Chicago, Illinois, USA).

\section{RESULTS}

In our COP study a total of 199 children were found to have an OAHI between 1 and 5 . Owing to limited resources, only the first 56 consecutive children were invited to participate in this follow-up study. Eleven (20\%) refused to return for repeat assessment owing to school commitments or other personal reasons. Children who were not invited to participate in the study were significantly older (10.6 (1.7) years vs 9.3 (1.7) years, $\mathrm{p}<0.001$ ), taller (141 (11) cm vs 134 (11) cm, p<0.001) and had a lower OAHI (1.9 (1.2-2.8) vs $2.2(1.5-2.9), p=0.037)$ than those who were approached. The former group also had a smaller proportion of subjects with habitual snoring $(21.7 \%$ vs $60.7 \%, p<0.001)$ and large tonsils $(16.3 \%$ vs $30.4 \%, p=0.027)$. No significant differences were found between the participants in the follow-up study and those who refused to participate.

\section{Subject characteristics at baseline and follow-up}

For the whole group, BMI and waist circumference increased significantly with age but their $z$-scores did not change significantly. The percentage of slow wave sleep decreased with increasing age. The OAHI values differed significantly between the two time points (fig 1). The arousal index was also significantly higher at follow-up. Neither tonsil nor adenoid size changed significantly during the 2-year follow-up period (see table 1 in online supplement).

For OSA-related symptoms, only 43 subjects were included in the analysis because 2 subjects did not return their questionnaire at follow-up. The prevalence of OSA-related symptoms did not change significantly except for habitual snoring which decreased from $67.4 \%$ to $46.5 \%(p=0.012)$.

\section{Comparison between worsened OSA group and referent group}

Thirteen of the 45 children (29\%) had worsened OSA. The increase in waist circumference was significantly greater in the worsened group than in the referent group (8.1 (6.3) vs 2.8 (5.5), $p=0.008$, table 1$)$. No significant differences were found in the presence of atopic diseases, history of adenotonsillitis in the follow-up period, household smoking and socioeconomic factors between the two groups (see table 2 in online supplement). 


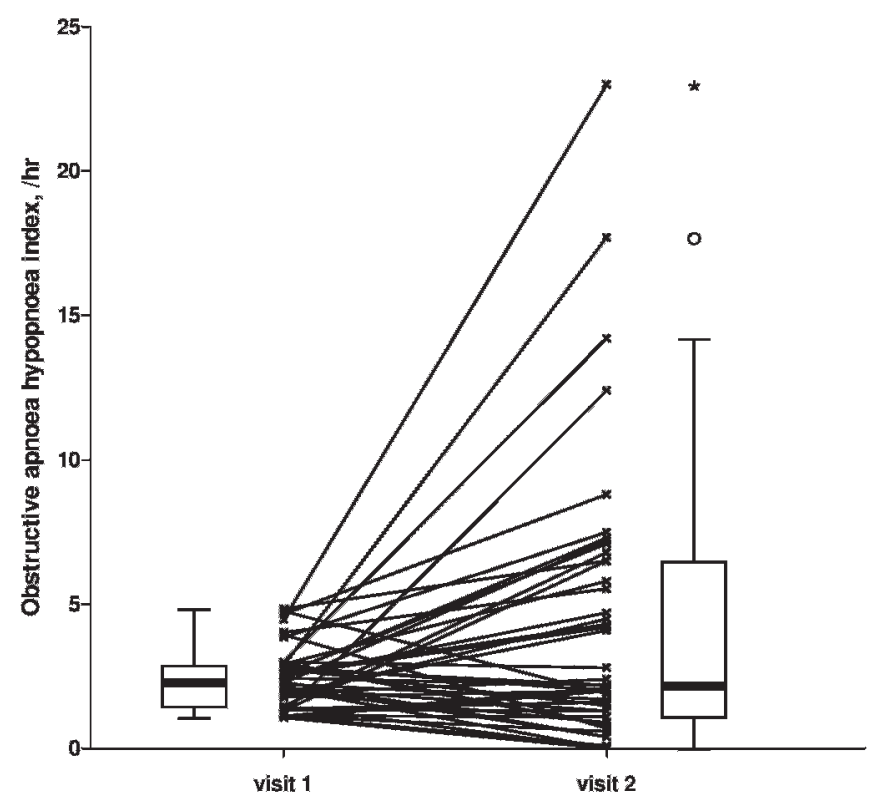

Figure 1 Change in obstructive apnoea-hypopnoea index of each subject over 2-year follow-up period.

The group with worsened OSA had a greater proportion of children with large tonsils at both baseline $(p=0.016)$ and follow-up ( $p=0.001$, table 2 ). The presence of large tonsils at baseline had a positive predictive value (PPV) of $53 \%$ and a negative predictive value (NPV) of $83 \%$ for worsening of OSA. Further analyses revealed that the worsened group had a lower prevalence of persistently small tonsils (31\% vs $78 \%$ ) and a higher prevalence of persistently large tonsils ( $46 \%$ vs $6 \%$ ) than the referent group $(p=0.003)$ (see table 3 in online supplement).

The group with worsened OSA had a higher prevalence of habitual snoring both at baseline $(p=0.008)$ and at follow-up $(p<0.001$, table 2$)$. The proportion of subjects with night sweating and restless sleep tended to decrease in the referent group ( $p=0.063$ and $p=0.070$, respectively) but not in the worsened OSA group (see table 4 in online supplement).

\section{Association between change in $\mathrm{OAHI}$ and baseline characteristics}

Several baseline variables including BMI z-score $(p=0.048)$, presence of large tonsils $(p=0.007)$ and presence of habitual snoring ( $p=0.045)$ were significantly associated with change in OAHI in univariate analysis (table 3). However, in a multivariate model adjusted for age and gender, only the presence of large tonsils had a significant effect $(p=0.001)$. Both age $(p=0.009)$ and male gender $(p<0.001)$ were also significant in the model (table 3 , model 1).

\section{Association between change in $\mathrm{OAHI}$ and change in other characteristics}

In univariate analysis, the change in OAHI was significantly associated with the presence of persistent large tonsils $(p<0.001)$, the change in waist circumference $(p<0.001)$ and its $z$-score $(p=0.028)$. The first two variables were then analysed together in a multivariate model with adjustment for age and gender. The change in waist circumference $z$-score was not included to avoid multicollinearity. Both factors together
Table 1 Demographic, clinical and socioeconomic features of referent group and group with worsened obstructive sleep apnoea (OSA)

\begin{tabular}{|c|c|c|c|}
\hline & $\begin{array}{l}\text { Referent } \\
(\mathrm{n}=32)\end{array}$ & $\begin{array}{l}\text { Worsened OSA } \\
(n=13)\end{array}$ & p Value \\
\hline Male gender, $\mathrm{n}(\%)$ & $21(65.6)$ & $10(76.9)$ & 0.724 \\
\hline \multicolumn{4}{|l|}{ Age, years } \\
\hline Baseline & $9.4(1.7)$ & $8.7(1.7)$ & 0.187 \\
\hline Change & $1.87(0.16)^{*}$ & $1.93(0.17)^{*}$ & 0.245 \\
\hline \multicolumn{4}{|c|}{ Body mass index z-score } \\
\hline Baseline & $0.82(0.98)$ & $0.87(1.11)$ & 0.892 \\
\hline Change & $0.03(0.44)$ & $0.20(0.50)$ & 0.271 \\
\hline \multicolumn{4}{|c|}{ Waist circumference z-score } \\
\hline Baseline & $0.67(1.17)$ & $0.83(0.80)$ & 0.653 \\
\hline Change & $0.06(0.59)$ & $0.37(0.60)^{*}$ & 0.123 \\
\hline
\end{tabular}

Values are mean (SD) unless stated otherwise.

${ }^{*}$ Significant change from baseline, $\mathrm{p}<0.05$ (paired $t$ test).

with male gender were significant predictors in the multivariate model (table 3 , model 2).

\section{DISCUSSION}

In this population-based cohort, mild OSA in about one-third of cases progressed to a more severe state after 2 years. Gender, large tonsils and increase in waist circumference were factors associated with disease progression. In particular, the presence of large tonsils had a PPV of $53 \%$ and an NPV of $83 \%$ for worsening OSA over a 2 -year period. To our knowledge, this is the first longitudinal study to identify factors associated with the natural progression of mild childhood OSA.

Previous studies showed that the majority of children with sleep-related breathing problems did not progress with time. ${ }^{11-13}$ In contrast, the present study found a substantial proportion of subjects $(29 \%)$ had worsening of OSA over a 2-year period. The discrepancy can probably be attributed to different characteristics of the study population and the definition of deterioration.

Identifying predictors for progression of OSA is important for clinicians. It allows better treatment prioritisation and a more evidence-based approach in patient management. In adults, weight gain and BMI at baseline were found to be significant predictors of worsening of OSA. ${ }^{23-25}$ However, the aetiology of OSA is quite different in adults and children, with upper airway enlarged lymphoid tissue being an important cause in children. So far, none of the published paediatric studies has examined for predictors of OSA progression. One previous study attempted to look for predictors of persistent snoring. Low maternal education, household smoking and snoring loudness were found to be significant predictors. ${ }^{10}$ However, the study was limited by its questionnaire-based design. Our current study found that household smoking and socioeconomic factors were not associated with worsening of OSA. In contrast, the presence of large tonsils and an increase in waist circumference were significant predictors for disease progression.

It is well established that adenotonsillar hypertrophy is a major cause of childhood OSA. We have previously reported that the tonsillar pharyngeal ratio, a radiological method for assessing tonsillar size, correlated positively with OSA severity. ${ }^{26}$ The positive association between the presence of large tonsils and worsening of OSA in this study raised the question whether children with mild OSA who have large tonsils should be considered for early surgical intervention. One could argue that such drastic action may not be necessary as tonsil size usually decreases with increasing age. In this cohort, 8 of the 15 
Table 2 Obstructive sleep apnoea (OSA)-related signs and symptoms of the two groups

\begin{tabular}{|c|c|c|c|}
\hline & $\begin{array}{l}\text { Referent } \\
\text { (n= 32) }\end{array}$ & $\begin{array}{l}\text { Worsened OSA } \\
(n=13)\end{array}$ & p Value \\
\hline \multicolumn{4}{|l|}{ OAHI (/h) } \\
\hline Baseline & $2.2(1.4-2.8)$ & $2.6(1.6-3.4)$ & 0.2 \\
\hline Follow-up & $1.7(0.7-2.4)$ & $7.3(6.7-13.3)$ & $<0.001$ \\
\hline Change & $-0.5(-1.4-0.8)$ & $4.9(3.9-11.2)$ & $<0.001$ \\
\hline \multicolumn{4}{|c|}{ Large tonsils, n (\%) } \\
\hline Baseline & $7(21.9)$ & $8(61.5)$ & 0.016 \\
\hline Follow-up & $2(6.3)$ & $7(53.8)$ & 0.001 \\
\hline $\mathrm{p}$ Value ${ }^{*}$ & 0.063 & 1.0 & \\
\hline \multicolumn{4}{|c|}{ Large adenoid, $n(\%)(n=40)$} \\
\hline Baseline & $5(18.5)$ & $4(30.8)$ & 0.4 \\
\hline Follow-up & $3(11.1)$ & $4(30.8)$ & 0.2 \\
\hline p Value ${ }^{*}$ & 0.7 & 1.0 & \\
\hline \multicolumn{4}{|c|}{ Habitual snoring, $n(\%)(n=43)$} \\
\hline Baseline & $18(56.3)$ & $11(100)$ & 0.008 \\
\hline Follow-up & $10(31.3)$ & $10(90.9)$ & $<0.001$ \\
\hline p Value ${ }^{*}$ & 0.021 & 1.0 & \\
\hline
\end{tabular}

Data shown as median (interquartile range) or mean (SD).

* Obtained from McNemar tests.

OAHI, obstructive apnoea hypopnoea index.

subjects (53.3\%) with large tonsils at baseline had persistently large tonsils 2 years later. Children with persistently large tonsils tended to be younger than those whose tonsils had subsequently decreased in size (age at baseline 7.9 (0.9) years vs 9.9 (2.1) years, $p=0.056)$. A previous study suggested that tonsil size peaked in 4-8-year-old children and the prevalence of tonsillar hypertrophy was low after the age of 8 years. ${ }^{27}$ However, longitudinal data describing the change in tonsil size in childhood are lacking and we do not know how long it takes for large tonsils to shrink. Taking into account important complications of OSA, regular surveillance is recommended to allow early detection of disease progression in children with mild OSA.
Increase in waist circumference, which outweighed BMI as a surrogate marker of weight gain, was found to be another predictor of OSA progression. Waist circumference may be a better predictor of cardiovascular risks than BMI. ${ }^{28}$ Furthermore, waist circumference has also been found to have a greater association with OSA than BMI. ${ }^{30}$ Increase in waist circumference, which signifies an increase in central obesity, can lead to lung volume reduction. The result is a loss of tracheal traction on the upper airway which then has a greater tendency to collapse. ${ }^{31}{ }^{32}$ Even though the waist circumference z-score was not different between those with OSA progression and those without, the marked increase in waist circumference in cases with disease progression further emphasises the need to implement weight control as part of the management of childhood OSA

Boys were more likely to have disease progression. Further analysis showed that girls were younger than boys (8.3 (1.7) years vs 9.7 (1.6) years at baseline, $p=0.012$ ), but there were no significant gender differences in weight gain and change in tonsil size. Both oestrogen and progesterone were found to be protective against upper airway collapse. ${ }^{33}$ However, most of the girls in this cohort were still prepubertal at follow-up. Further studies are needed to delineate the underlying mechanism for this gender difference.

Our data showed poor correlation between OAHI-defined disease progression and OSA-related symptoms. This was not surprising because a high OAHI is not always accompanied by symptoms. The Wisconsin sleep cohort reported a minimal OSA prevalence rate of $2 \%$ and $4 \%$ for males and females when daytime complaints were included as one of the diagnostic criteria. The rate would inflate greatly to $9 \%$ and $24 \%$ if symptoms were excluded in the definition. ${ }^{34}$ Similarly, in our COP study in which the definition of childhood OSA was based on ICSD II criteria, ${ }^{35}$ the prevalence was $4.8 \%$ (95\% CI $4.3 \%$ to $5.4 \%)$. When the definition was based solely on OAHI $\geqslant 1$, the prevalence increased dramatically to $40.3 \%$ (95\% CI $39.1 \%$ to 41.5\%). ${ }^{14}$ Thus, symptom-based diagnostic criteria may not

Table 3 Linear regression analyses assessing the association between the change in $\mathrm{OAHI}$ and different independent variables $(n=45)$

\begin{tabular}{|c|c|c|c|c|c|c|}
\hline & \multirow{2}{*}{\multicolumn{2}{|c|}{ Univariate }} & \multicolumn{4}{|l|}{ Multivariate } \\
\hline & & & \multicolumn{2}{|l|}{ Model 1* } & \multicolumn{2}{|l|}{ Model $2 \dagger$} \\
\hline & $\beta$ (SE) & p Value & $\beta$ (SE) & p Value & $\beta$ (SE) & p Value \\
\hline Male gender & $2.10(1.44)$ & 0.153 & $4.69(1.29)$ & $<0.001$ & $2.60(1.18)$ & 0.033 \\
\hline \multicolumn{7}{|l|}{ Baseline characteristics } \\
\hline Age & $-0.64(0.39)$ & 0.106 & $-0.92(0.34)$ & 0.009 & $-0.01(0.36)$ & 0.987 \\
\hline BMI & $0.26(0.18)$ & 0.174 & & & & \\
\hline BMI z-score & $1.33(0.66)$ & 0.048 & $0.97(0.55)$ & 0.084 & & \\
\hline Waist & $0.03(0.06)$ & 0.643 & & & & \\
\hline Waist z-score & $0.81(0.63)$ & 0.206 & & & & \\
\hline $\mathrm{OAHI}$ & $0.76(0.65)$ & 0.248 & & & & \\
\hline Large tonsils & $3.75(1.33)$ & 0.007 & $4.36(1.24)$ & 0.001 & & \\
\hline Habitual snoring & $2.85(1.38)$ & 0.045 & $0.92(1.21)$ & 0.453 & & \\
\hline \multicolumn{7}{|l|}{ Changes over 2 years } \\
\hline BMI & $0.51(0.45)$ & 0.261 & & & & \\
\hline BMI z-score & $0.44(1.50)$ & 0.769 & & & & \\
\hline Waist & $0.41(0.09)$ & $<0.001$ & & & $0.30(0.09)$ & 0.002 \\
\hline Waist z-score & $2.48(1.09)$ & 0.028 & & & & \\
\hline Persistent large tonsils & $6.26(1.51)$ & $<0.001$ & & & $5.69(1.36)$ & $<0.001$ \\
\hline
\end{tabular}


provide a true picture of OSA epidemiology. Furthermore, symptoms were based on parental reporting, which could be subject to recall bias.

This study has several limitations. First, subjects only underwent a single night PSG and there is a possibility of subject misclassification due to the first-night effect and nightto-night PSG variability. Our research group, however, has previously shown that a single night study can correctly identify $80 \%$ of all OSA cases and, as resources were limited, a single night was deemed to be adequate. ${ }^{21}$ Second, this study involved two visits across a relatively short follow-up period. A larger scale study with longer follow-up and more assessments at different time points would be invaluable. Although pubertal status was not assessed in the study, most of our subjects were likely to be in the prepubertal stage as reflected by their young mean age of 11.1 years. ${ }^{36}$ Finally, the study did not examine complications of childhood OSA such as cardiovascular and neurobehavioural outcomes. If these measures were also shown to deteriorate corresponding to worsening of OSA, clinicians would be more inclined to treat mild cases of OSA more aggressively.

We conclude that children, especially boys, with mild OSA and tonsillar hypertrophy should be closely monitored by regular follow-up to allow early detection of worsening OSA. Weight control is important in the management of childhood OSA.

Acknowledgements: The authors thank all the staff at the Sleep Assessment Unit of Shatin Hospital for their logistic support and the cooperation and participation of all the schools, children and their parents.

Funding: This study was supported by funding from the Research Grants Council of the Hong Kong Special Administrative Region, China (CUHK4161/02M).

Competing interests: None.

Ethics approval: Informed consent was obtained from parents or legal guardians and verbal assent was obtained from each child. The study was approved by the institutional ethical committee.

Provenance and peer review: Not commissioned; externally peer reviewed.

\section{REFERENCES}

1. Chan YS, Li AM, Au CT, et al. Cardiac remodelling and dysfunction in children with obstructive sleep apnoea: a community based study. Thorax 2009;64:233-9.

2. Li AM, Au CT, Sung RY, et al. Ambulatory blood pressure in children with obstructive sleep apnoea: a community based study. Thorax 2008;63:803-9.

3. Li AM, Chan MH, Chan DF, et al. Insulin and obstructive sleep apnea in obese Chinese children. Pediatr Pulmonol 2006;41:1175-81.

4. Gozal D, Capdevila OS, Kheirandish-Gozal L. Metabolic alterations and systemic inflammation in obstructive sleep apnea among nonobese and obese prepubertal children. Am J Respir Crit Care Med 2008;177:1142-9.

5. Halbower AC, Degaonkar M, Barker PB, et al. Childhood obstructive sleep apnea associates with neuropsychological deficits and neuronal brain injury. PLoS Med 2006; $\mathbf{3}: \mathrm{e} 301$

6. Tishler PV, Larkin EK, Schluchter MD, et al. Incidence of sleep-disordered breathing in an urban adult population: the relative importance of risk factors in the development of sleep-disordered breathing. JAMA 2003;289:2230-7.

7. Redline S, Schluchter MD, Larkin EK, et al. Predictors of longitudinal change in sleepdisordered breathing in a nonclinic population. Sleep 2003;26:703-9.
8. Newman AB, Foster G, Givelber R, et al. Progression and regression of sleepdisordered breathing with changes in weight: the Sleep Heart Health Study. Arch Intern Med 2005;165:2408-13.

9. Ali NJ, Pitson D, Stradling JR. Natural history of snoring and related behaviour problems between the ages of 4 and 7 years. Arch Dis Child 1994;71:74-6.

10. Urschitz MS, Guenther A, Eitner S, et al. Risk factors and natural history of habitual snoring. Chest 2004;126:790-800.

11. Marcus CL, Hamer A, Loughlin GM. Natural history of primary snoring in children Pediatr Pulmonol 1998;26:6-11.

12. Topol HI, Brooks LJ. Follow-up of primary snoring in children. J Pediatr 2001;138:291-3.

13. Anuntaseree W, Kuasirikul S, Suntornlohanakul S. Natural history of snoring and obstructive sleep apnea in Thai school-age children. Pediatr Pulmonol 2005:39:415-20.

14. Li AM, So HK, Au CT, et al. Epidemiology of obstructive sleep apnea syndrome in Hong Kong Chinese children: a two-phase study. Abstract presented at Hong Kong Paediatric Society Annual Scientific Meeting, Hong Kong, November 2008.

15. Li AM, Cheung A, Chan D, et al. Validation of a questionnaire instrument for prediction of obstructive sleep apnea in Hong Kong Chinese children. Pediatr Pulmonol 2006; 41:1153-60.

16. Sung RY, So HK, Choi KC, et al. Waist circumference and waist-to-height ratio of Hong Kong Chinese children. BMC Public Health 2008:8:324

17. Leung SS, Cole TJ, Tse LY, et al. Body mass index reference curves for Chinese children. Ann Hum Biol 1998;25:169-74.

18. Wing YK, Hui SH, Pak WM, et al. A controlled study of sleep related disordered breathing in obese children. Arch Dis Child 2003;88:1043-7.

19. American Thoracic Society. Cardiorespiratory sleep studies in children: establishment of normative data and polysomnographic predictors of morbidity. Am J Respir Crit Care Med 1999;160:1381-7.

20. Sleep Disorders Atlas Task Force. EEG arousals: scoring and rules and examples. Sleep 1992;15:173-84.

21. Li AM, Wing YK, Cheung A, et al. Is a 2-night polysomnographic study necessary in childhood sleep-related disordered breathing? Chest 2004;126:1467-72.

22. Bland JM, Altman DG. Measurement error. BMJ 1996;313:744

23. Tishler PV, Larkin EK, Schluchter MD, et al. Incidence of sleep-disordered breathing in an urban adult population: the relative importance of risk factors in the development of sleep-disordered breathing. JAMA 2003;289:2230-7.

24. Redline S, Schluchter MD, Larkin EK, et al. Predictors of longitudinal change in sleepdisordered breathing in a nonclinic population. Sleep 2003;26:703-9.

25. Newman AB, Foster G, Givelber R, et al. Progression and regression of sleepdisordered breathing with changes in weight: the Sleep Heart Health Study. Arch Intern Med 2005;165:2408-13.

26. Li AM, Wong E, Kew J, et al. Use of tonsil size in the evaluation of obstructive sleep apnoea. Arch Dis Child 2002;87:156-9.

27. Akcay A, Kara CO, Dagdeviren E, et al. Variation in tonsil size in 4- to 17-year-old schoolchildren. J Otolaryngol 2006:35:270-4.

28. Sung RY, Yu CC, Choi KC, et al. Waist circumference and body mass index in Chinese children: cutoff values for predicting cardiovascular risk factors. Int $\mathrm{J}$ Obes (Lond) 2007;31:550-8.

29. Watts K, Bell LM, Byrne SM, et al. Waist circumference predicts cardiovascular risk in young Australian children. J Paediatr Child Health 2008;44:709-15.

30. Grunstein R, Wilcox I, Yang TS, et al. Snoring and sleep apnoea in men: association with central obesity and hypertension. Int J Obes Relat Metab Disord 1993;17:533-40.

31. Schwab RJ, Gupta KB, Gefter WB, et al. Upper airway and soft tissue anatomy in normal subjects and patients with sleep-disordered breathing: significance of the lateral pharyngeal walls. Am J Respir Crit Care Med 1995:152:1673-89.

32. Van de Graaff WB. Thoracic traction on the trachea: mechanisms and magnitude. J Appl Physiol 1991;70:1328-36.

33. Cistulli PA, Barnes DJ, Grunstein RR, et al. Effect of short term hormone replacement in the treatment of obstructive sleep apnoea in postmenopausal women. Thorax 1994;49:699-702.

34. Young T, Palta M, Dempsey J, et al. The occurrence of sleep-disordered breathing among middle-aged adults. N Eng/ J Med 1993;328:1230-5.

35. American Academy of Sleep Medicine. International classification of sleep disorders. Diagnostic and coding manual. 2nd edn. Westchester, Illinois: American Academy of Sleep Medicine, 2005.

36. Lam TH, Shi HJ, Ho LM, et al. Timing of pubertal maturation and heterosexual behaviour among Hong Kong Chinese adolescents. Arch Sex Behav 2002;31:359-66. 